\title{
Diagnosis of COVID-19 Infected Lungs from Chest X-ray Images using Deep Learning Algorithms
}

\author{
Ta-seen Reaz Niloy and Md. Abdur Rahman
}

\begin{abstract}
Severe Acute Respiratory Symptom Coronavirus 2 (SARS-CoV-2) is newly discovered as a beta coronavirus. The virus-induced unexplained etiological pneumonia and is referred to as the 2019 Coronavirus Disease (COVID-19). Though the disease has appeared in a new way, there is no medication for transited patients. So, for diagnosing the COVID-19 infected lungs from $X$-Ray images, an automated technique has been suggested in this manuscript. The proposed system is divided into two stages: Image Acquisition and Selection of Algorithms. In the IAA, the training data's size has been increased by augmenting the image in different ways. The Algorithm Selection portion explained the Convolutional Neural Network (CNN) and VGG19. The Tuning of hyperparameters section determines the precise hyperparameter combination in order to maximize the model's performance. In this study, CNN and VGG19 are used and found accuracy scores of $97 \%$ and $67 \%$, respectively. The comparative analysis shows that the propound method acts better than the solution that exists. Eventually, Precision, Recall, and F1 score have been extracted and interpreted the model's loss functions in the research. This research has carried out by focusing on essential aspects in terms of COVID-19. Therefore, for the diagnosis of coronavirus infection, the technique can be used effectively.
\end{abstract}

Index Terms-Deep Learning, Image Augmentation, COVID19 Lung Damage, Image Classification, VGG19, and CNN.

\section{INTRODUCTION}

$\mathrm{C}$ ORONA virus disease (COVID-19) is one of today's most talked-about conditions that first emerged in late 2019 in Wuhan, China, and soon transmitted worldwide. Since this difficulty is an infectious one, the phenomenon has afflicted peoples from all countries, and the mortality rate is the highest rather than any other diseases that occurred beforehand. The virus is new, and the medication for unwellness is being created by scientists worldwide. There are no precise medications available to stop or cure COVID-19 as of now. Therefore, the case of efficient detection methods is a

\section{Ta-seen Reaz Niloy}

Dept. of Electrical and Electronic Engineering (EEE)

American International University Bangladesh.

Email: taseenreazniloy3@gmail.com

Md. Abdur Rahman

Dept. of Electrical and Electronic Engineering (EEE)

American International University Bangladesh.

Email: arahman@ aiub.edu breakthrough in minimizing the outcome of unwellness by early detection and isolation. With the advancement of medical imaging and power-assisted computational recognition, most diseases can be diagnosed effectively. Image comprehension requires the identification of irregularities, the assessment of their places and boundaries, and the calculation of their scale and severity. Human experts' scarce availability and fatigue, high consultancy fees, and rough estimation procedures restrict image interpretation efficacy. Also, the square calculation of shapes, positions, and structures in medical abnormalities is highly variable. Even for specialist physicians, this makes diagnosing difficult. Usually, therefore, human specialists feel a need to support resources to understand medical images correctly. Radiological imaging is another important diagnostic method for COVID-19. In CT images, most COVID-19 cases have early stages of groundglass opacity and later pulmonary consolidation. Rare instances have been identified with rounded morphology and peripheral lung distribution. [1]. The images for various viral types of pneumonia are similar even though standard CT images may help diagnose at an early stage of suspect cases. They imbricate with other lung disorders that are infectious or inflammatory. Consequently, COVID-19 is difficult for radiologists to distinguish from other viral pneumonia. This is the motivation of this proposed study to build a deep learning model for the clinical aspect that can make diagnosis effectively.

Deep learning (DL) is a collection of machine learning approaches applied in various applications [2]. A significant advantage of deep learning over ancient techniques is that a complex feature extraction component is unnecessary. The network takes the raw input and maps it to the output that is defined. With no manual intervention, the characteristics are mechanically learned by the neural network [3]. With an increased number of hidden layers, deep learning models are associated with conventional networks' uplift. The hidden layers dramatically increase the efficiency of those networks. Convolution Neural Networks (CNN) are commonly used in computer vision [4]. Various medical imaging researchers have also used CNN for image processing operations to feel segmentation and classification [5]. Despite CNN's promised accuracy, it is widely used for image identification and 
recognition. The $\mathrm{CNN}$ uses a hierarchical architecture to create a network, similar to a funnel that eventually provides a fully linked layer with all of the neurons combined. This is our main motto for creating a COVID-19 diagnosis and screening model from Chest X-ray images using modern computation such as the deep learning algorithm. In this report, a pipeline for medical image processing and an efficient approach to diagnosing the disease has been established. Three contributions can be found in this proposed study.

- Acute disease such as COVID-19 is identified using deep learning algorithms of considerable well-being to physicians.

- In addition to the multi-layer neural network, the pretrained model VGG19 and the Convolution Neural Network (CNN) has been used to identify COVID-19 accurately with a comparison of existing works in terms of accuracy.

- The pipeline of medical image classification is shown, and it is designed to be implemented in real life by creating a robust model through the optimization of the loss function.

\section{LITERATURE REVIEW}

The following research has been performed on COVID-19 detection over the year using X-ray or CT scan-based images. The paper [2] proposed a DL-based architecture to detect COVID-19 that performed efficiently with $90 \%$ and $100 \%$ accuracy, respectively. The authors of the study utilized the publicly available covid-chest X-ray-dataset and conducted their primary research. The screening of COVID-19 using Chest X-ray images was carried out utilizing a Deep Learning (DL) based approach in paper [3]. The authors used domain extension transfer learning (DETL) with the pre-trained model on a similar massive chest X-ray dataset. They eventually achieved a $90.3 \%$ accuracy rate. Deep feature extraction was performed using pre-trained models (ResNet18, ResNet50, ResNet101, VGG16, and VGG19) in [4]. The acquirement of the fine-tuned ResNet50 model was found to be $92.6 \%$, while the end-to-end training of the evolved CNN model yielded a 91.6\% efficiency. The paper [5] suggested a DL based model for COVID-19 pattern recognition from X-ray images that were both effective and successful. They also exploit with a hierarchical classifier the underlying taxonomy of the problem. The findings indicate that the method proposed was capable of producing a high-quality model with a $93.9 \%$ overall Precision. The authors of the study [6] trained multiple deep convolutional networks: regular, pneumonia, and COVID-19, based on two open-source datasets, and they eventually obtained $91.4 \%$ of accuracy. A CNN-based model for diagnosing COVID-19 diseases from the X-ray images was proposed in [7] and achieved $92.74 \%$ accuracy. Another research [8] used Chest X-ray images to create a prediction model with $97.3 \%$ accuracy on Random Forest and $97.7 \%$ accuracy found after applying XGBoost.

The above studies [2-8] were conducted based on the COVID19 diagnosis using Chest X-ray images with the deep neural network. The accuracy of some research is relatively low, and the benefits of image preprocessing and data augmentation are not described in detail in the above literature review. These studies performed on the traditional CNN algorithm; no customize or hyper-parameters tuning are found in the above benchmark.

In this proposed research, the identification of COVID-19 infected lungs from X-Ray images has been conducted using pre-trained models, for example, VGG19 (19 layers deep) and CNN that are incredibly optimized for the proposed model's loss function, making a robust model in terms of clinical aspect. Utilizing a pre-trained model requires less preparation and less effort in constructing the model architecture, and it is substantially more accurate than applying a traditional CNN approach [9]. The image augmentation pipeline has been demonstrated for enhancing the training images to reduce the overfitting, positively impacting the research community.

\section{RESEARCH METHODOLOGY}

The Proposed Research Methodology (PRM) is divided into two distinctive sections: Image Acquisition and Algorithm Selection. The area on Image Acquisition is further subdivided into four stages: Research Dataset, Image Augmentation Approach (IAA), Training Method and Tuning of hyperparameters. The dataset, mentioned in detail in the Research Dataset section, is used in this analysis. The IAA portion explained how the dataset was improved by generating a modified version of the current training set. The Algorithm Selection section finally delineated CNN and VGG19. The conceptual visualization of the proposed system and the entire design from the acquisition of images to the X-ray images classification is visually presented in Fig. 1

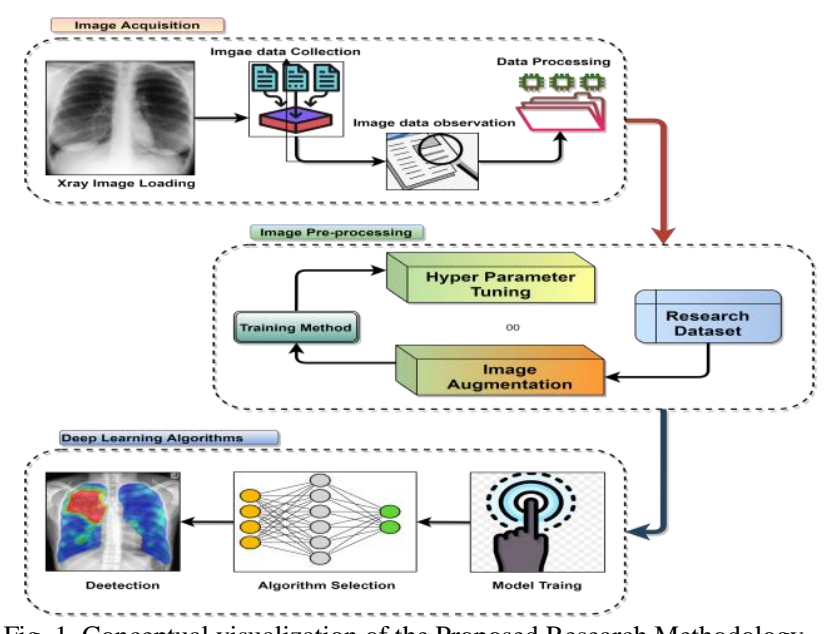

Fig. 1. Conceptual visualization of the Proposed Research Methodology 


\section{IMAGE ACQUISITION}

This section deals with the datasets that are used in this study and the image augmentation approach. This research's primary objective is to identify COVID-19 infected lungs from the XRay image dataset, so a publicly accessible dataset has been used. The batch size is considered to have 16 and image height and width 500 X 500 pixels. While images must be resized to a fixed size before being fed into the $\mathrm{CNN}$, it needs to be resized before applying to the algorithm. [10]. If the size of the dimension is 500 or greater, and the batch size is greater than 16 , the system can crash because the RAM is used up (Google Colab free trial version is about $13 \mathrm{~GB}$ ). One alternative to consider is a smaller dimension size with a larger batch size [11]. The batch size of 16 indicates that 16 samples of the training dataset will be performed to estimate the error gradient before the model weight is updated. For many factors, batch size 16 was selected in this case; for example, small batch sizes make it easier to fit one batch of training data into memory (e.g., when using a GPU) [12].

\section{A. Research Dataset}

The publicly available dataset was collected from the online repository [13-15]. This dataset consists of COVID-19 infected images and is divided into Train and Test sets. However, for this study, we divided the images into two categories: COVID-19 POSITIVE (also known as class 0) and COVID-19 NEGATIVE (also known as class 1). The following figures (Fig.2 \& Fig.3) visualize the dataset.
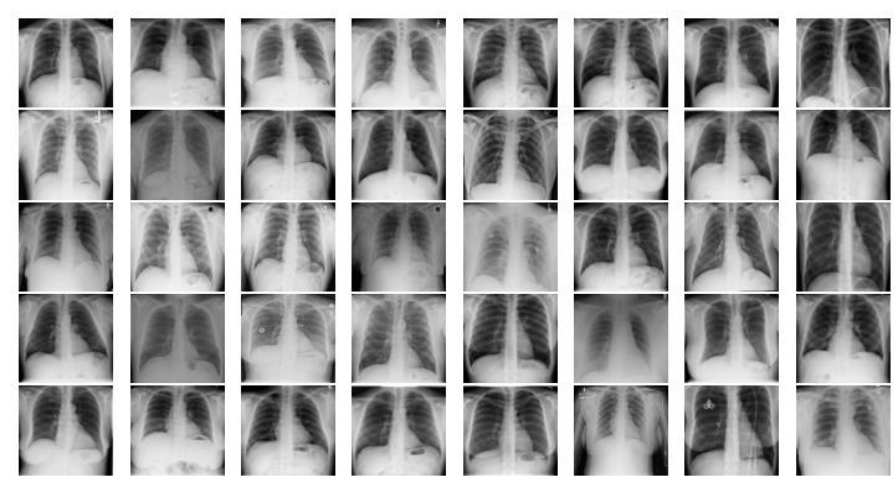

Fig. 2. Chest X-ray images of COVID-19 negative cases [15]
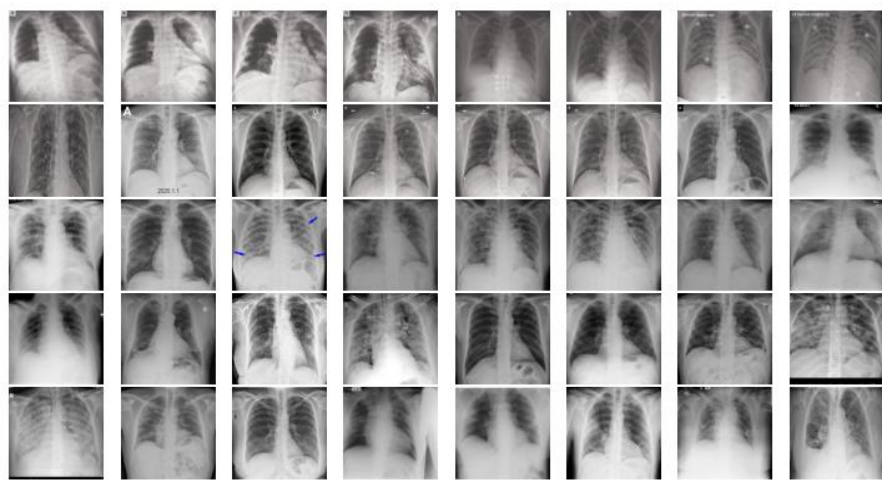

Fig. 3. Chest X-ray images of COVID-19 positive cases [16]

\section{B. Image Augmentation Approach (IAA)}

Image Augmentation increases the dataset's size by creating a modified version of the current training set's images to maximize the dataset's variance and eventually improve the model's ability to predict new images. Deep networks require vast quantities of training data to achieve fair effectiveness [17]. Image augmentation is usually needed to create a robust image classifier with a small data size to ameliorate the deep network's representation. [18]. Image augmentation creates training images artificially using a variety of processing method or combinations of multiple processing. [19]. In this case of IAA, rotation (45 degrees, 135 degrees, 225 degrees, 315 degrees), twists, shear, and flips (Flip left-right, Flip updown, Flip up-down-left-right), contrast, saturation are performed. Fig. 4, 5, 6, and 7 demonstrate the IAA that we have carried out on our dataset.

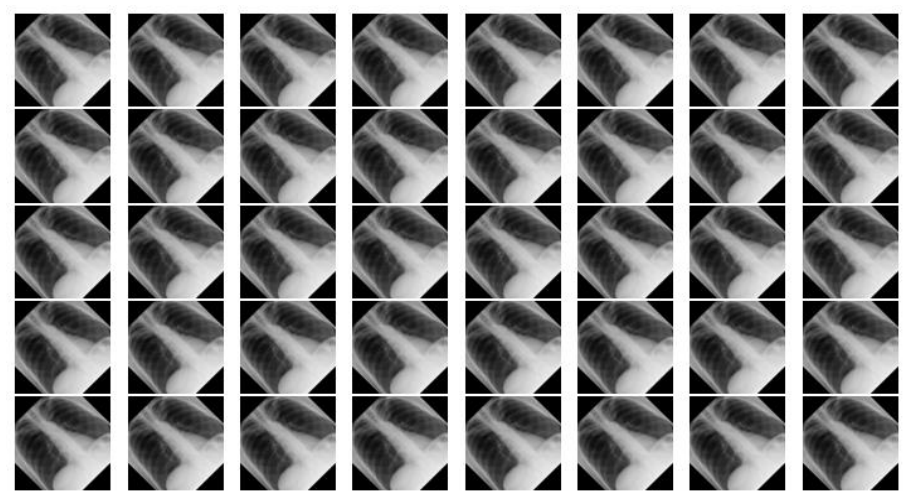

Fig. 4. Image Augmentation (rotate 45 degrees)

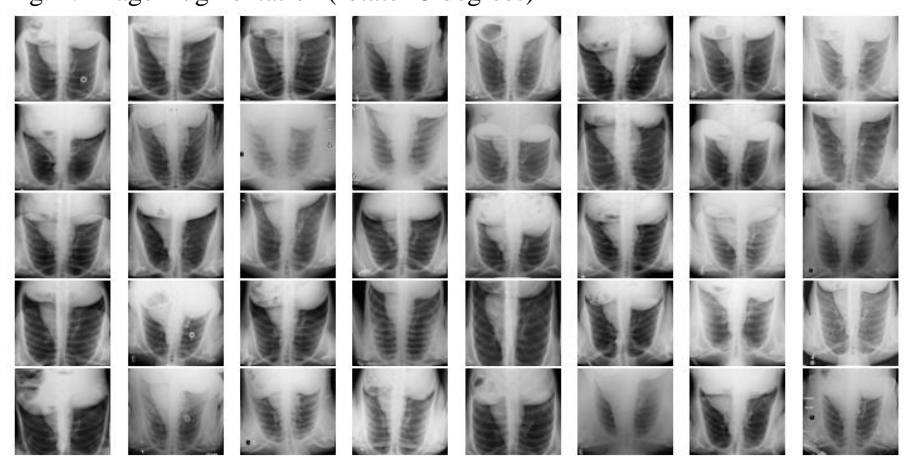

Fig. 5. Image Augmentation (Flip up-down-left-right)
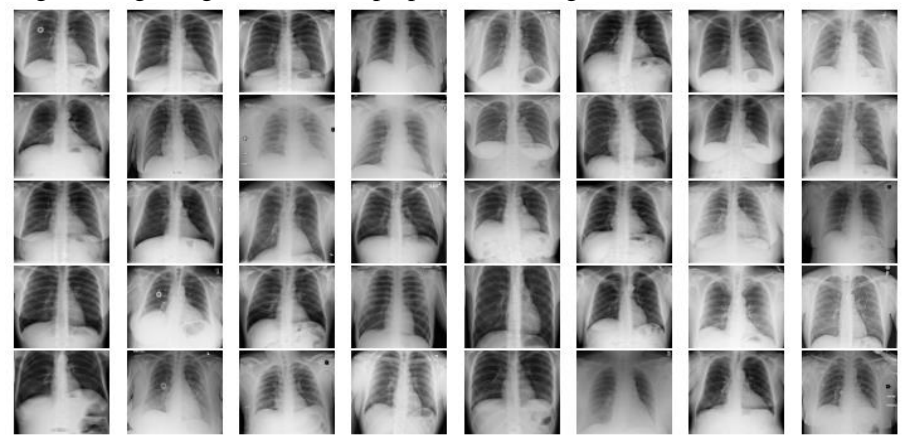

Fig. 6: Image Augmentation (Adjusted saturation) 


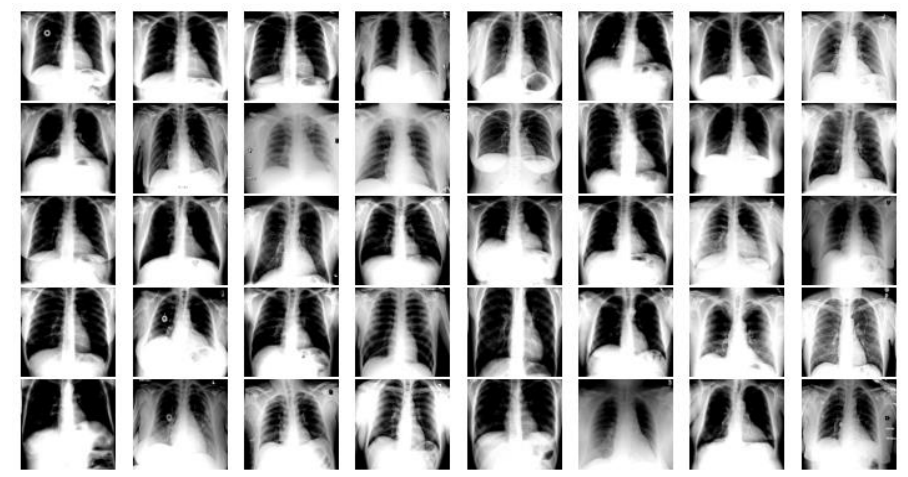

Fig. 7: Image Augmentation (Adjusted contrast)

\section{TRAINING METHOD}

The optimization algorithm minimizes the loss function by changing the network weights. The binary cross-entropy loss function is used in the proposed model $(\mathrm{CNN})$. It is used for binary classification problems to produce probabilities over the ' $n$ ' number of classes for each image. Data Augmentation is used to resolve the imbalanced dataset problem [18]. With an increase in data, training data is improved to boost model training. Initially, training images are rescaled, which reduces the size of the image. Images are created by rotating them within a given range. Adam, a replacement optimization algorithm for stochastic gradient descent for training deep learning models, plays an essential role in rapid conversion and efficiency enhancement, including customized weight loss [20]. After the related experiments, the batch size was set to 16. We used 100 epochs to train and test our deep neural network. There is no standard process to select the batch size and epochs to the best of our knowledge.

To put it another way, it is desirable to increase the number of epochs and training based on the error rates. An epoch is a single learning period during which the learner sees the entire training data collection [21]. The models' performance is evaluated using different evaluation metrics, such as F1 score, Precision, Validation Accuracy, Sensitivity, and Specificity, etc., which are detailed interpreted in the results section.

\section{TUNING THE HYPER-PARAMETERS}

This section demonstrates hyperparameters' effect in the training procedure and our proposed system's evaluation phase. In the experiments, the image size for input was initially $500 \times 500$ pixels. The input size was then reduced to change the network and boost efficiency. By changing the number of convolution layers, we have found that the number of ideal separable convolution layers is 12 , and the typical convolution layers are 6.

\section{ALGORITHM SELECTION}

The medical imaging area has overgrown in the last few decades. The development of automated clinical decision- making approaches has received broad support from the medical community. Thus, in this section, an automated method based on Convolution Neural Networks (CNN) and VGG19 are interpreted to diagnose COVID-19 using chest Xray images. In this section, the architecture of $\mathrm{CNN}$ and VGG19 are introduced.

\section{A. VGG19 Architecture}

VGG19 is a Transfer learning model which consists of 19 layers [3]. Transfer learning is commonly used to train new models with a range of sources that aren't linked to the most recent training model but are similar and have restricted target data. Using minimal target data to train models, migrating parameters to a new model decreases training time and increases accuracy. Transfer learning is used in this study to detect COVID-19 contaminated lungs based on these advantages [22].

\section{B. Convolutional Neural Network (CNN)}

The Convolutional neural network (CNN) is a form of multilayer neural network designed to identify visual patterns from pixel images that have been minimally preprocessed. CNN is made up of two primary components: convolutional layers and pooling layers. [23]. It is prevalent, and the best part about CNN is that no need to extract the features. The model learns to extract features by convolution images and filters to construct invariant features passed on to the next layer. To build more invariant and abstract elements, the next layer's components are complicated with various filters [24].

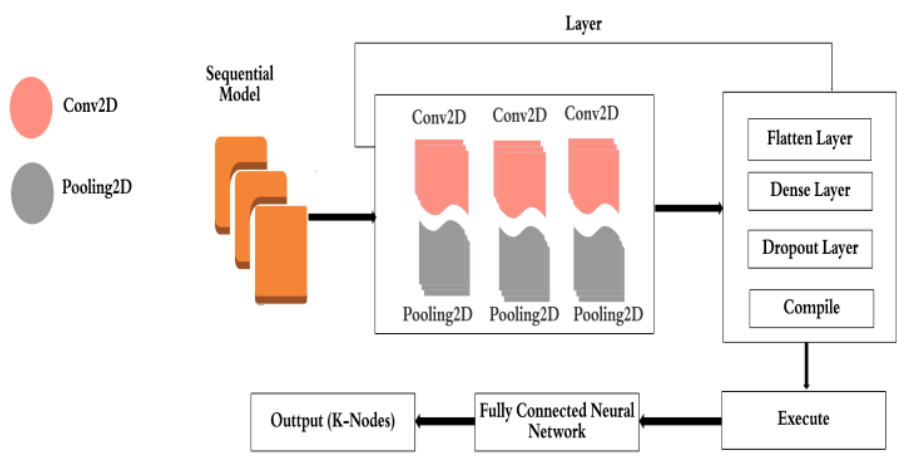

Fig. 8. The architecture of the Proposed CNN

Towards medical image classification, the CNN-based model is widely applied [24]. CNN is a substantial feature extractor and can prevent costly and challenging feature engineering by identifying medical images. As a consequence of its high Precision, CNNs are used for image classification and recognition. The $\mathrm{CNN}$ follows a hierarchical model that, like a funnel, operates on building a network and eventually provides an entirely connected layer where all the neurons are linked, and the output is processed. CNN blends input data with learned features and uses 2D convolutional layers, making this design well suited to $2 \mathrm{D}$ data processing, such as images. Since the need for manual feature extraction is 
removed by CNNs, it is not required to choose the features necessary to identify the images.

Nevertheless, to make use of a pre-trained model. If the amount of image data does not have to be abundant, overfitting occurs in the model [25]. On the other hand, CNN can be implemented smoothly to find medical images with limited data [26].

\section{RESULT \& ANALYSIS}

The Results Analysis section has been divided into three parts: Empirical Consequence Report (ECP), Report of Model Evaluation (RME), and Comparative Study. The classification report of CNN and VGG19 are depicted in the ECP section. The loss function optimization between CNN and VGG19 is shown in the ECP.

\section{A. Empirical Consequence Report (ECP)}

Two techniques are used to evaluate a classification model: the confusion matrix and the classification report. The confusion matrix abridges a classification algorithm; it consists of four values: True Positive (TP), False Positive (FP), True Negative (TN), and False Negative (FN) [27]. To assess the accuracy of a classification algorithm's predictions, a classification report is used. This research was followed by equations (1), (2), (3), and (4) to determine the Precision, Recall, and F1 score and the classification report is also listed in Table I [28].

Precision: The ratio of the model's true positive estimate to the total (correct and incorrect) positive assessment.

It is articulated as-

Precision $=\frac{T P}{T P+F P}$

Recall / Sensitivity: The ratio of being able to predict as positive. It is given in mathematical form as-

Recall $=\frac{T P}{T P+F N}$

F1 score: This is the harmonic mean of Precision and Recall, which gives a more accurate calculation than the accuracy Metric of incorrectly categorized instances. Mathematically, it's a given. It is shown mathematically-

$$
\mathrm{F} 1=\frac{2 . \text { Precision } \cdot \operatorname{Re} \text { call }}{\text { Precision }+ \text { Recall }}
$$

Accuracy: It is the measure of all the instances correctly predicted. It is given as-

$$
\text { Accuracy }=\frac{T P+T N}{T P+T N+F P+F N}
$$

Table I shows the classification metrics Precision, Recall and F1 score on a per-class basis where the Precision has been written "P" and the same way the Recall is "R" and the F1 score is "F1". The metrics are calculated by using true and false positives, true and false negatives. In contrast, CNN and VGG19 have been implemented in this proposed study, so the two models' classification reports are interpreted separately in Table I. Since this research indicates binary classification and has experimented with classifying positive and negative case (COVID-19) infected lungs, the Precision, Recall, F1 score, and Accuracy for each case was calculated through equations 1,2,3 and 4. Fig. 9 illustrates how the model is accurately classified.

TABLE I

CLASSIFICATION REPORT OF PROPOSED CNN AND VGG19 MODEL.

\begin{tabular}{|c|c|c|c|c|c|c|c|}
\hline \multirow{2}{*}{ Model } & \multicolumn{3}{|c|}{ COVID-19 (Negative) } & \multicolumn{3}{|c|}{ COVID-19 (Positive) } \\
\cline { 2 - 8 } & $\mathbf{P}$ & $\mathbf{R}$ & $\mathbf{F 1}$ & $\mathbf{P}$ & $\mathbf{R}$ & $\mathbf{F 1}$ & Accuracy \\
\hline CNN & 0.95 & 1.00 & 0.98 & 1.00 & 0.95 & $\begin{array}{c}0.9 \\
7\end{array}$ & 0.97 \\
\hline VGG19 & 0.88 & 0.37 & 0.52 & 0.90 & 0.95 & $\begin{array}{c}0.7 \\
5\end{array}$ & 0.67 \\
\hline
\end{tabular}

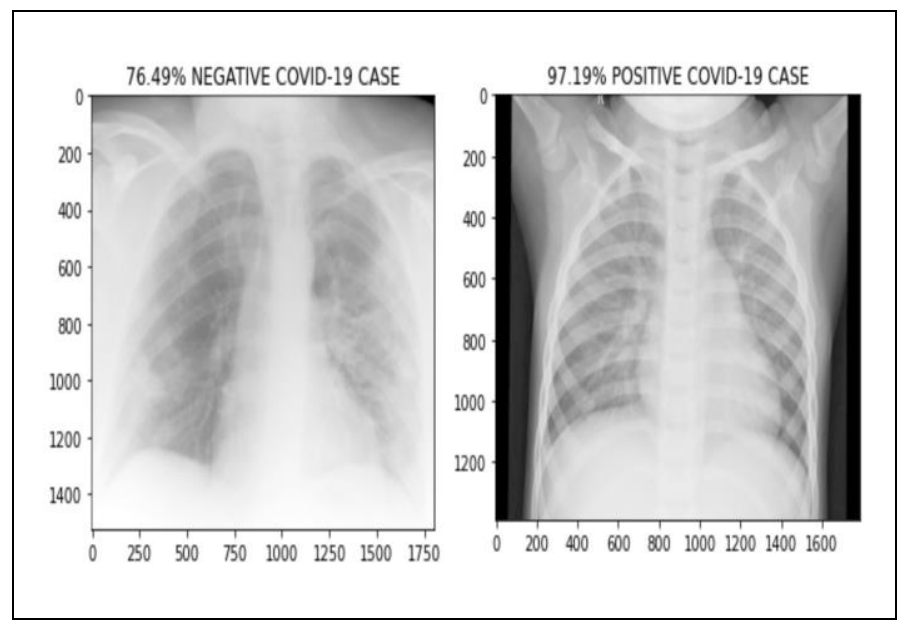

(a)

(b)

Fig. 9. (a) Detecting COVID-19 negative case with $76.49 \%$ accuracy (b) Classifying COVID-19 positive case with $97.19 \%$ accuracy.

By looking at Fig. 9. It can be explicitly observed that the proposed $\mathrm{CNN}$ model accurately predicts the positive and negative classes separately from X-ray images. 


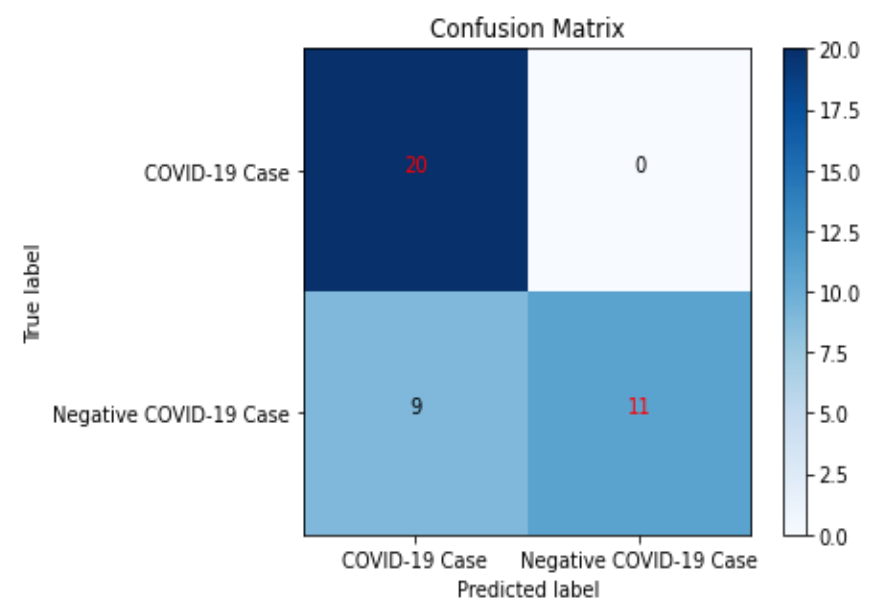

Fig. 10. Confusion matrix above COVID-19 positive and negative classes.

A confusion matrix is a technique to summarize the outcomes of a classification algorithm. If there is an unequal number of findings in each class or more than two groups in the dataset, the classification itself's accuracy may be misleading. To visualize significant predictive analytics such as Recall, specificity, accuracy, and Precision, confusion matrices are used. Fig.10 represents the Confusion matrix for the CNN model. The different values of the Confusion matrix would be as follows:

- True Positive $(\mathrm{TP})=20$, indicating the model correctly categorized 20 positive class data points.

- True Negative $(\mathrm{TN})=11$; specifying the model appropriately categorized 11 data points from the negative class.

- $\quad$ False Positive $(\mathrm{FP})=0$; this means that the model wrongly counted 0 negative class data points as positive class data.

- $\quad$ False Negative $(\mathrm{FN})=9 ; 9$; the data points of the 9 positive classes were incorrectly classified by the model as belonging to the negative category.

\section{B. Report of the Model Evaluation (RME)}

This section demonstrates the comparison between the CNN and VGG19 models in terms of their loss and accuracy. Since there is some loss in training and testing during the model build because no model can predict $100 \%$ accurately, this research aimed to optimize the loss and ensure sensitivity and specificity. Fig. 11-14 shows the loss function optimization based on the VGG19 and CNN. Learning good values for all the weights and the bias from labelled examples is all that training a model entails [29]. Loss, on the other hand, is the outcome of a poor forecast. A loss is a number that reflects how incorrect the model's prediction was for a single example [29]. Training a model is to find a collection of weights and biases that have a low loss across all instances on average. For every model, the higher the loss (wrong prediction), the worse it is [30]. The loss is measured for both training and validation, and it is used to determine how well the models perform.
The following diagrams represent the losses \& accuracy of two different models (CNN and VGG19). The details sequence and consequence are shown in Fig. 11, Fig.12, Fig.13, and Fig.14, respectively.

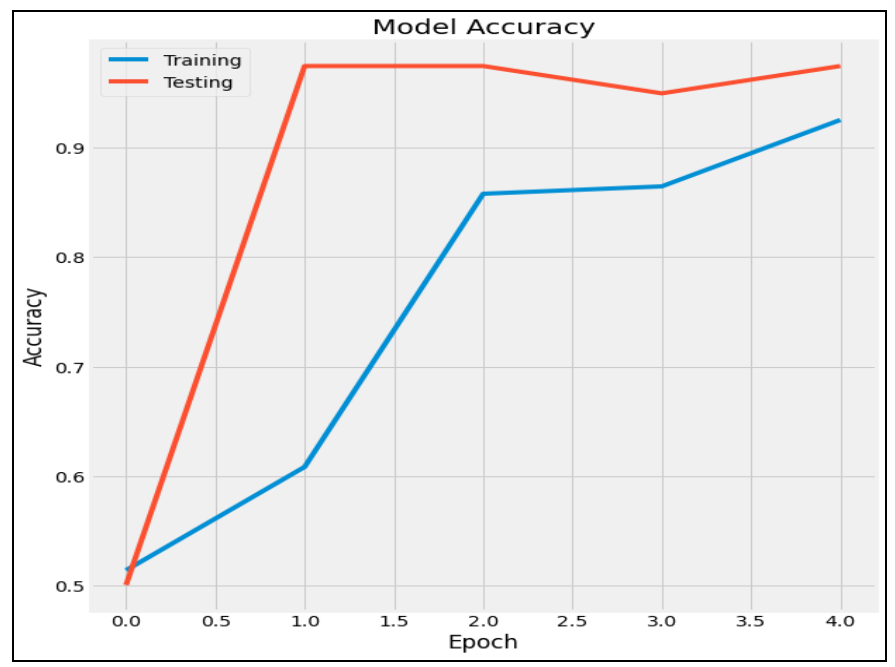

Fig. 11. The representation of the proposed CNN's accuracy.

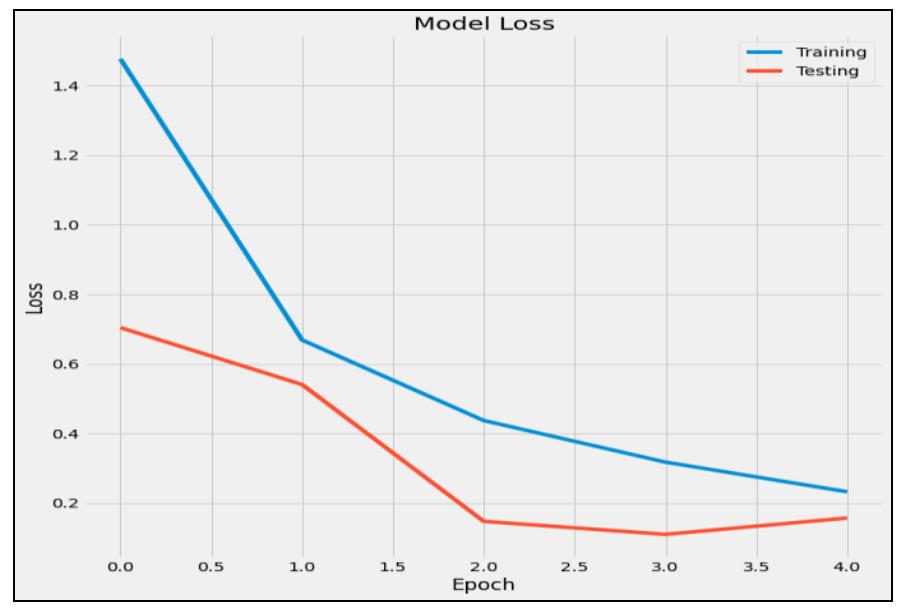

Fig. 12. Visualizing the loss optimization of the $\mathrm{CNN}$.

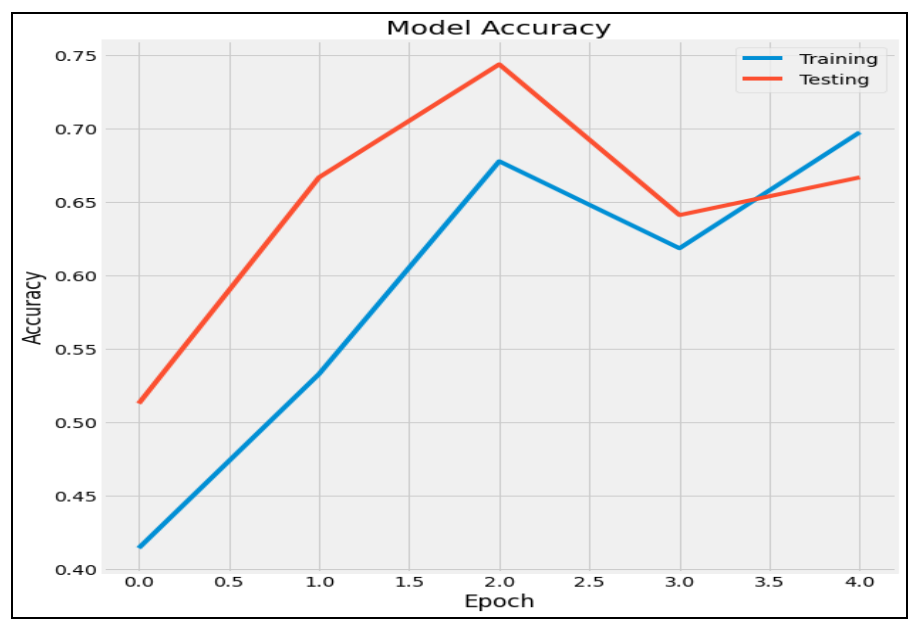

Fig. 13. Visualizing the accuracy of the proposed VGG19. 


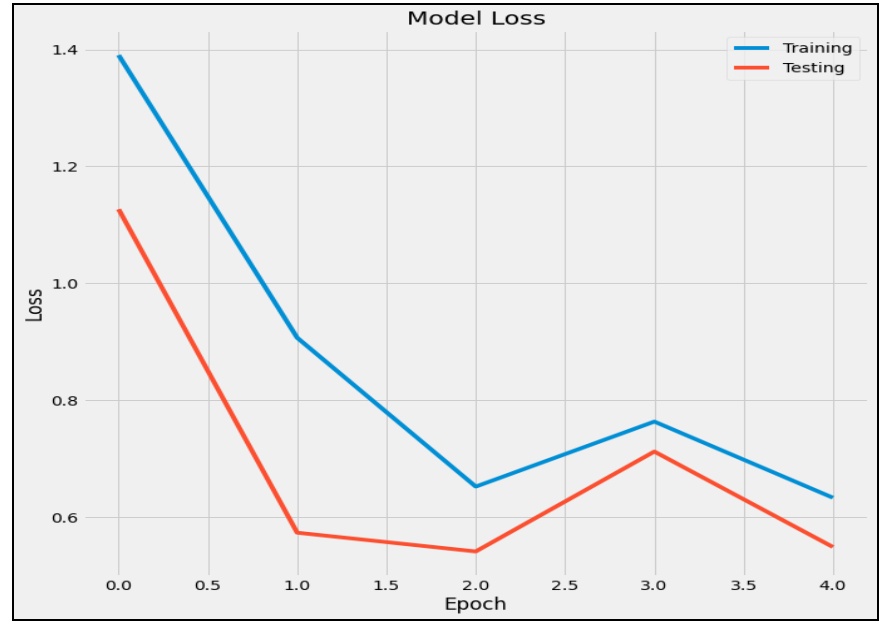

Fig. 14. Graphical presentation of the model's (VGG19) loss.

CNN's findings can be described as interpretations as this research has found satisfactory accuracy of CNN. It is clear from Fig. 11 that the upward trend of accuracy was observed when the epoch began from 0 to 1.0. After that, when the epoch remained steady at 2.0 , there was some downward trend of accuracy. Thus, when the epoch reaches 4.0, the accuracy experienced a rapid surge. On the other hand, the line graph (Fig.12) explained the relationship between the epoch and the model's loss. Test and training data start at 0 and go down to 1.0 and 2.0; their losses begin to plunge. When the epoch is 4.0, the loss function dramatically declined. In Fig.13, the epoch starts at 0 and continues to rise and stays unchanged at 2.0 , but suddenly, the accuracy decreases a bit when the epoch goes to 3.0. Since then, there has been significant growth in accuracy between 3.5 and 4.0. Lastly, Fig.14 explains the interconnection of accuracy with the loss of the model (VGG19). The amount of loss was much higher initially, and the loss decreased dramatically in epoch 1.0. Then between 1.5 and 2.0, the rate of loss gradually decreases and stabilizes at 2.0. The ratio of loss is peaked at 3.0, and then it slightly goes down to 4.0. The accuracy plots (Fig. 11 and 12) show that the model should be trained a little more because both dataset's accuracy trend has been rising over the last few epochs. If these parallel plots begin to diverge consistently, it might discontinue training at an earlier epoch.

\section{Comparative Study}

This section compares our proposed research with the current study explicitly developed to diagnose and screen the COVID19 infected lungs. Table II shows comparative analysis based on our proposed model in terms of accuracy. In the context of Table II, it can be said that previous research has accomplished the task of detecting COVID-19 using a variety of algorithms. As in the literature review, it is mentioned that the data augmentation and preprocessing are not carried out perfectly in the previous studies. On the other hand, in our proposed model, the data augmentation has been conducted ideally with the hyperparameter tuning that enhances the training data's size for making a robust model with reasonable accuracy.
TABLE II

COMPARATIVE STUDY OF THE PROPOSED RESEARCH

\begin{tabular}{|l|l|}
\hline Proposed Method & Performance Accuracy \\
\hline CheXNet [6] & $90.5 \%$ \\
\hline Transfer learning (DETL) [7] & $90.13 \%$ \\
\hline CNN [8] & $91.6 \%$ \\
\hline Deep artificial neural network [9] & $93.9 \%$ \\
\hline Xception and ResNet50V2 [10] & $91.4 \%$ \\
\hline CNN [11] & $92.74 \%$ \\
\hline Deep learning [12] & $97 \%$ \\
\hline CNN [19] & $96.69 \%$ \\
\hline This proposed work (CNN) & $97 \%$ \\
\hline
\end{tabular}

\section{CONCLUSION \& FUTURE WORK}

This research's objective has been achieved by applying the model effectively to assist clinicians in diagnosing the disease. The model automates the process of identifying intricate medical image patterns at a level comparable to veteran radiologists. The expected approaches are evaluated and measured via an experiment. The various metrics are calculated, such as Accuracy, Precision, Sensitivity, Specificity, F1 score. Compared to the opposite approaches, the proposed method has shown critical progress. On CNN, the accuracy reached $97 \%$. Therefore, the planned methodology for detecting coronavirus disease (COVID-19) is used, particularly for lung damage from chest X-ray images.

\section{REFERENCES}

C. Huang, Y. Wang, X. Li, L. Ren, J. Zhao, Y. Hu, et al., "Clinical features of patients infected with 2019 novel coronavirus in Wuhan, China," The lancet, vol. 395, pp. 497-506, 2020.

A. Mangal, S. Kalia, H. Rajgopal, K. Rangarajan, V. Namboodiri, S. Banerjee, et al., "CovidAID: COVID-19 detection using chest X-ray," arXiv preprint arXiv:2004.09803, 2020.

S. Basu, S. Mitra, and N. Saha, "Deep learning for screening covid-19 using chest x-ray images," in 2020 IEEE Symposium Series on Computational Intelligence (SSCI), 2020, pp. 2521-2527. A. M. Ismael and A. Şengür, "Deep learning approaches for COVID-19 detection based on chest X-ray images," Expert Systems with Applications, vol. 164, p. 114054, 2021.

E. Luz, P. L. Silva, R. Silva, L. Silva, G. Moreira, and D. Menotti, "Towards an effective and efficient deep learning model for covid19 patterns detection in $\mathrm{x}$-ray images," arXiv preprint arXiv:2004.05717, 2020.

M. Rahimzadeh and A. Attar, "A new modified deep convolutional neural network for detecting COVID-19 from X-ray images,"

M. Gour and S. Jain, "Stacked convolutional neural network for diagnosis of covid-19 disease from $\mathrm{x}$-ray images," arXiv preprint arXiv:2006.13817, 2020.

R. Kumar, R. Arora, V. Bansal, V. J. Sahayasheela, H. Buckchash, J. Imran, et al., "Accurate prediction of COVID-19 using chest xray images through deep feature learning model with smote and machine learning classifiers," MedRxiv, 2020.

M. Dąbrowski and T. Michalik, "How effective is Transfer Learning method for image classification," in Position papers of the Federated Conference on Computer Science and Information Systems, 2017, pp. 3-9. arXiv preprint arXiv:2004.08052, 2020. 
[10] M. Hashemi, "Web page classification: a survey of perspectives, gaps, and future directions," Multimedia Tools and Applications, pp. $1-25,2020$

[11] H. Deshmukh, "Medical X-ray Image Classification using Convolutional Neural Network," vol. 2021, ed, 2020.

[12] M. L. Mastery, "How to Control the Stability of Training Neural Networks With the Batch Size," vol. 2021, ed, 2020.

[13] J. P. C. a. P. M. a. L. Dao, "COVID-19 image data collection," arXiv, 2020.

[14] RSM, "COVID-19 DATABASE," ed.

[15] P. Mooney, "Chest X-Ray Images (Pneumonia)," ed.

[16] A. W. Linda Wang, Zhong Qiu Lin, Paul McInnis, Audrey Chung, Hayden Gunraj, James Lee, Matt Ross, Blake VanBerlo, Ashkan Ebadi, Kim-Ann Git, Abdul Al-Haimi, "Figure 1 COVID-19 Chest X-ray Dataset Initiative," ed.

[17] N. Koleva, "When and When Not to Use Deep Learning," vol. 2021, ed. data iku, 2020.

[18] C. Shorten and T. M. Khoshgoftaar, "A survey on image data augmentation for deep learning," Journal of Big Data, vol. 6, pp. $1-48,2019$

[19] S. Ghosh, N. Das, I. Das, and U. Maulik, "Understanding deep learning techniques for image segmentation," ACM Computing Surveys (CSUR), vol. 52, pp. 1-35, 2019.

[20] D. P. Kingma and J. Ba, "Adam: A method for stochastic optimization," arXiv preprint arXiv:1412.6980, 2014.

[21] S. SHARMA, "Epoch vs Batch Size vs Iterations," vol. 2021, ed, 2017.

[22] J. Zhou, X. Yang, L. Zhang, S. Shao, and G. Bian, "Multisignal VGG19 Network with Transposed Convolution for Rotating Machinery Fault Diagnosis Based on Deep Transfer Learning," Shock and Vibration, vol. 2020, 2020.

[23] P. Dutta, P. Upadhyay, M. De, and R. Khalkar, "Medical image analysis using deep convolutional neural networks: Cnn architectures and transfer learning," in 2020 International Conference on Inventive Computation Technologies (ICICT), 2020, pp. 175-180.

[24] Q. Li, W. Cai, X. Wang, Y. Zhou, D. D. Feng, and M. Chen, "Medical image classification with convolutional neural network," in 2014 13th international conference on control automation robotics \& vision (ICARCV), 2014, pp. 844-848.

[25] D. Hendrycks, K. Lee, and M. Mazeika, "Using pre-training can improve model robustness and uncertainty," in International Conference on Machine Learning, 2019, pp. 2712-2721.

[26] Ž. Emeršič, D. Štepec, V. Struc, and P. Peer, "Training convolutional neural networks with limited training data for ear recognition in the wild," arXiv preprint arXiv:1711.09952, 2017.

[27] S. Visa, B. Ramsay, A. L. Ralescu, and E. Van Der Knaap, "Confusion Matrix-based Feature Selection," MAICS, vol. 710, pp. 120-127, 2011.

[28] K. P. Shung, "Accuracy, Precision, Recall or F1?," vol. 2021, ed, 2018.

[29] Google, "Descending into ML: Training and Loss," vol. 2021, ed.

[30] intellipaat, "How to interpret "loss" and "accuracy" for a machine learning model," vol. 2021, ed.
Honor for six consecutive times (from Spring 2017- to Fall 2019). Besides, she has completed an international internship from AIESEC in Cairo University in a solar energy project with a partnership with the company named "E-Solar Renewable Energy". She has good experience in machine learning, deep learning and antenna design. She is currently researching biomedical image classification and machine learning.

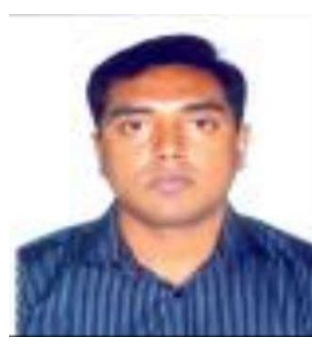

Md. Abdur Rahman, experienced Researcher with a demonstrated history of working in the higher education industry. He has extensive knowledge of Radio Frequency (RF), Wireless communication, Biomedical Imaging, Machine Learning and Antenna propagation. Profound research professional with Doctor of Philosophy $(\mathrm{PhD})$ from Tokyo Institute of Technology University in 2013 focused on Software Defined Radio (SDR) and has experience as a Postdoctoral Researcher in Federation University Australia from 2014, focused on Application of modern signal processing techniques in gene expressions to form gene regulatory network (GRN). His research interest is focused on RF/Microwave Circuits and Systems, Digital Signal Processing, Biomedical Image processing, Nanoelectronics, Machine Learning, Wireless communication, Antenna propagation. Currently, he is working as an Associate Dean in the Faculty of Engineering at American International University-Bangladesh (AIUB) and a professor in EEE, AIUB.

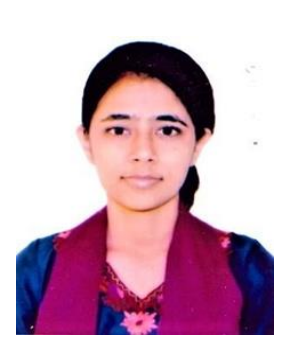

Ta-seen Reaz Niloy received her undergraduate degree in Electrical and Electronic Engineering (EEE) from American International University Bangladesh (AIUB). At present, she is working as a Teaching Assistant (TA) under the Faculty of Engineering (FE) at American International UniversityBangladesh (AIUB). She has received MAGNA CUM LAUDE (Academic Award Based on CGPA) on the 19th Convocation Ceremony and the Deans Award on Capstone Project (2nd Position) based on her undergrad thesis. During her undergrad period, she has received Deans' List 